\title{
Anti-IL-1 alpha Monoclonal Antibody MABp1
}

National Cancer Institute

\section{Source}

National Cancer Institute. Anti-IL-1 alpha Monoclonal Antibody MABp1. NCI Thesaurus.

Code C88325.

A human IgG1 monoclonal antibody directed against interleukin-1 alpha (IL1a) with potential A human IgG1 monoclonal antibody targ eting the inflammatory cytokine interleukin-1 alpha (IL1a) with potential antineoplastic, anti-cachectic and anti-angiogenic activities. Anti-IL1a monoclonal antibody MABp1 targets and binds to IL1a and prevents IL1a activity. This prevents IL1a-mediated tumorigenesis and angiogenesis. In addition, MABp1 abrogates IL1a-mediated cachexia. IL1a, an inflammatory mediator expressed on monocytes, platelets and overexpressed by certain tumors, plays a key role in the promotion of tumor cell growth, metastasis and invasion. In addition, IL1a stimulates metabolic activity in the central nervous system. 